\title{
Efficacy of Open Debridement and Polyethylene Exchange in Strictly Selected Patients with Infection after Total Knee Arthroplasty
}

\author{
Whee Sung Son, $\mathrm{MD}^{1}$, Oog-Jin Shon, $\mathrm{MD}^{1}$, Dong-Chul Lee, $\mathrm{MD}^{2}$, Sang-Jin Park, $\mathrm{MD}^{3}$, and Han Seok Yang, $\mathrm{MD}^{1}$ \\ ${ }^{1}$ Department of Orthopedic Surgery, Yeungnam University Medical Center, Daegu; ${ }^{2}$ Department of Orthopedic Surgery, Bo Gang Hospital, Daegu; ${ }^{3}$ Department of \\ Anesthesia, Yeungnam University Medical Center, Daegu, Korea
}

\begin{abstract}
Purpose: To assess the efficacy of open debridement and polyethylene exchange (ODPE) combined with proper antibiotic therapy in strictly selected patients with infection after total knee arthroplasty (TKA) and analyze factors associated with treatment failure.

Materials and Methods: From January 2010 to January 2014, 25 cases that underwent ODPE under the diagnosis of infection within four weeks after TKA or acute hematogenous infection within five days of symptom onset were reviewed in this study.

Results: Treatment was successful in 22 out of 25 cases (88.0\%). Factors associated with failure were accompanying infection (periprosthetic infection in the ipsilateral foot, cervical parotid abscess, and masticator space abscess) and diagnosis of rheumatoid arthritis (RA) before TKA. Resistant bacteria did not entail a risk. On clinical results, the mean postoperative Lysholm score and Korean Knee score were 81.4 and 79.4, respectively, the knee range of motion was $115.4^{\circ} \pm 12.9^{\circ}$, and duration of hospitalization was $32.3 \pm 8.4$ days. On radiographic results, $3.47 \pm 1.56 \mathrm{~mm}$ joint line elevation and a valgus change of $0.61^{\circ} \pm 2.35^{\circ}$ in knee alignment were observed.

Conclusions: ODPE combined with appropriate antibiotics therapy could be a useful treatment method for infection after TKA if the procedure is performed within a symptom duration of five days or less in the absence of accompanying infection in patients whose indication for TKA was not RA.
\end{abstract}

Keywords: Knee, Arthroplasty, Infection, Debridement

\section{Introduction}

Infection after total knee arthroplasty (TKA) is a potentially fatal condition that may lead to a number of complications and it is not easy to cure. The increasing frequency of TKA in the growing elderly population has resulted in a rise in the incidence of postoperative infection ${ }^{1,2}$. Infection following TKA can be categorized by the Tsukayama classification as infection with culture

Received July 14, 2016; Revised (1st) October 16, 2016;

(2nd) December 21, 2016; Accepted January 2, 2017

Correspondence to: Oog-Jin Shon, MD

Department of Orthopaedic Surgery, Yeungnam University Medical

Center, 170 Hyeonchung-ro, Nam-gu, Daegu 42415, Korea

Tel: +82-53-620-3640, Fax: +82-53-628-4020

E-mail: ossoj@med.yu.ac.kr

This is an Open Access article distributed under the terms of the Creative Commons Attribution Non-Commercial License (http://creativecommons.org/licenses/by-nc/4.0/) which permits unrestricted non-commercial use, distribution, and reproduction in any medium, provided the original work is properly cited. positive during revision arthroplasty (type I), early postoperative infection (type II), acute hematogenous infection (type III), and late chronic infection (type IV). Of these, type II and III infections are known to occur within four postoperative weeks and considered as indications for open debridement and polyethylene exchange (ODPE) ${ }^{3,4)}$.

However, outcomes of ODPE in type II and III infections vary widely among studies. Successful outcomes of ODPE were reported by Gardner et al. ${ }^{5)}$ in 2011 in 11 out of 21 cases (43\%) with a mean symptom duration of 8.4 days (range, 2 to 28 days); Cobo et al. ${ }^{6}$ in 2011 in 67 out of 117 cases (57.3\%) within 30 days after TKA; Holmberg et al. ${ }^{7)}$ in 2015 in 109 out of 145 cases (75\%) with a mean symptom duration of 4 days (range, 1 to 32 days) and within an average of 23 days (range, 2 to 357 days) after TKA; and Koh et al. ${ }^{8}$ in 2015 in 32 out of 52 cases (71\%) with a mean symptom duration of 8 days (range, 4 to 26 days) for Tuskayma type III and within 8 days (range, 6 to 80 days) after TKA for Tsukayma type II. Schoifet and Morrey ${ }^{9)}$ stated that the success 
rate of ODPE was strongly associated with the period between symptom expression and the procedure in Tsukayama type III infection and with the period between TKA and the procedure in Tsukayama type II infection. Some researchers reported particularly low success rates of ODPE performed within four weeks after symptom onset of Tsukayama type III infection despite it being the conventional indication for the procedure ${ }^{6,10,11)}$.

In this study, we hypothesized that it would be possible to obtain a good outcome of ODPE in Tsukayma type III infection cases by using a shorter duration of symptoms as an indication than the conventional criterion of within four weeks. Thus, we performed ODPE within five days after the onset of symptoms for Tsukayma type III infection whereas we strictly adhered to the existing criterion of four weeks for Tsukayma type II infection. The purpose of this study was to evaluate the success rate and usefulness of ODPE performed using these criteria, and identify factors associated with treatment failure.

\section{Materials and Methods}

\section{Materials}

Among patients who underwent TKA from January 2010 to January 2014, ODPE was performed in 25 cases (23 patients) 1) with acute hematogenous infection (Tsukayama type III infection) strictly within five days after the symptom onset, 2) with Tsukayama type II infection strictly within four weeks after TKA, and 3) with no radiological finding of prosthesis loosening or osteomyelitis. From 2010 to 2011, the diagnosis of infection was based on clinical symptoms with joint fluid analysis, erythrocyte sedimentation rate (ESR), and C-reactive protein (CRP) as suggested by Tsukayama et al. ${ }^{3}$. Since 2012, the criteria for peripros- thetic joint infection presented by the Musculoskeletal Infection Society in the United States have been used for the diagnosis of infection $^{12)}$ (Table 1).

The average patient age was 70.8 years (range, 35 to 86 years) and there were five males and eighteen females. Thirteen patients (56\%) had histories of immune-compromising comorbidities including diabetes mellitus, chronic kidney disease, and rheumatoid arthritis (RA). The underlying diseases were quantified using the Charlson comorbidity index (CCI), and the mean score was 3.75 points (range, 2 to 6 points). In four cases, the indication for TKA was identified as RA rather than osteoarthritis. The average follow-up period was 29.4 months (range, 24 to 35 months). The average body mass index was $26.52 \mathrm{~kg} / \mathrm{m}^{2}$. Ten cases were classified as Tsukayama type II infection that was identified at a mean of 17.4 days (range, 4 to 28 days) after TKA and 15 cases as Tsukayama type III infection with mean symptom duration of 3.3 days (range, 1 to 5 days).

This study was approved by the Institutional Review Board of the corresponding author's hospital (YUMC 2016-09-015).

\section{Surgical Technique and Antibiotic Treatment}

A midline skin incision was made along the previous incision line using an anteromedial approach to expose the infected joint. Wide debridement and irrigation were performed for all infected or necrotic tissues, followed by complete synovectomy to the posterior capsule with the polyethylene removed (Fig. 1). Bacteria from the synovial fluid, joint capsule, and synovial sheath were cultured intraoperatively. After irrigation performed using more than $10 \mathrm{~L}$ saline solution, the patient was surgically draped again, and all surgical staff had their gloves and gowns changed before using new instruments. The existing polyethylene was replaced

Table 1. Definition Criteria of Periprosthetic Joint Infection (PJI) Presented by the American Musculoskeletal Infection Society

PJI is considered present when one of the major criteria exists or four out of six minor criteria exist

Major criteria

(1) There is a sinus tract communicating with the prosthesis

(2) A pathogen is isolated by culture from at least two separate tissue or fluid samples obtained from the affected prosthetic joint

Minor criteria

(a) Elevated serum erythrocyte sedimentation rate and serum C-reactive protein concentration

(b) Elevated synovial leukocyte count

(c) Elevated synovial neutrophil percentage (polymorphonuclear leukocyte)

(d) Presence of purulence in the affected joint

(e) Isolation of a microorganism in one culture of periprosthetic tissue or fluid

(f) Greater than five neutrophils per high-power field in five high-power fields observed from histologic analysis of periprosthetic tissue at 9,400 magnification 

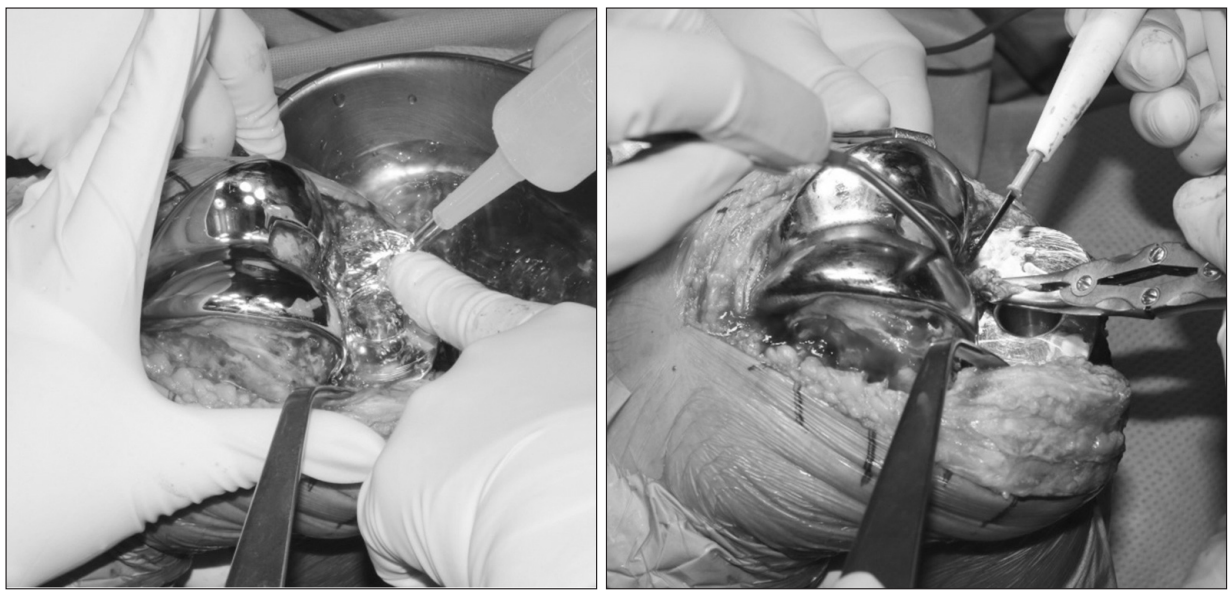

Fig. 1. Intraoperative photographs showing complete synovectomy to the posterior capsule with polyethylene removed.

by a thicker one ( $3.47 \mathrm{~mm}$ on average), and additional irrigation was performed. The procedure ended with insertion of drainage, followed by wound suture. The average surgery time was 104.58 minutes (range, 65 to 135 minutes), and drains were removed 3.91 days after the procedure on average (range, 1 to 10 days).

After consultation with the Infection Department of Internal Medicine, a broad spectrum antibiotic therapy was prescribed, and the antibiotics were changed according to the results of aspirated synovial fluid culture and intraoperative culture. Rifampin was used when Staphylococcus aureus was detected. In culturenegative cases, vancomycin and third generation cephalosporins that can control the gram-positive and negative bacteria were used.

All patients received intravenous antibiotics treatment for at least four weeks according to the general treatment protocol. If the results of serologic tests (ESR and CRP) and physical examination showed no evidence of further infection, the intravenous antibiotic therapy was ceased, and oral antibiotics were administered for two weeks. If it was determined that the patient did not respond to the prescribed antibiotics in serial blood tests and clinical examination, the antibiotics were changed to different types to which the isolated bacteria were sensitive. The average duration of the intravenous antibiotic therapy was 33.8 days (range, 28 to 46 days).

Postoperative protocol was slightly modified for each patient, but typically, knee range of motion exercises were started one week after surgery. After discharge, follow-ups were scheduled at two weeks, one month, three months, and six months, and then routinely once a year in the outpatient department. At each outpatient visit, the patient underwent clinical knee examination, ESR and serum CRP level tests, and radiographic examination, and they were checked for infection recurrence. If a recurrence was suspected, a joint fluid analysis was immediately performed for confirmation.

\section{Assessment and Analysis of Results}

Treatment failure was defined as: 1) patient death before the end of antibiotic treatment; 2) need for antibiotic therapy for continuous infection (elevated serum ESR, serum CRP, synovial leukocyte count, and neutrophil percentage, and maintained purulence in the affected joint); and 3) need for another surgery of debridement or two-stage revision arthroplasty due to infection recurrence.

To analyze risk factors for treatment failure, identified bacteria, history of accompanying diseases, infection status, and the amount of heterogenous materials (constrained type of component, rod, wedge, or other additional fixation materials) were analyzed. As possible covariates or confounders, patient age, CCI, preoperative diagnosis of RA and the results of primary blood test and joint aspiration test (ESR, CRP, synovial leukocyte count, and synovial neutrophil percentage) were investigated. To assess the efficacy of ODPE, the Lysholm score and the Korean Knee score (KKS) were examined at the final follow-up visit, and the range of motion of the knee joint and total duration of hospitalization were also assessed.

ODPE often requires more aggressive debridement since infection needs to be controlled by the one-stage procedure unlike in the two-stage revision arthroplasty. Since a loss of soft tissue balance following such aggressive debridement may result in an alignment change, postoperative alignment changes were measured. A modified Figgie's method was used to assess changes in the joint line and the method of the American Knee Society was used to assess changes in the joint alignment ${ }^{13}$. All clinical and radiological assessments were performed by the same researcher. Intergroup comparison was made using Mann-Whitney test at the $p<0.05$ significance level, and Fisher exact test and logistic 
regression analysis were carried out to analyze the factors associated with treatment failure (SPSS ver. 16.0; SPSS Inc., Chicago, IL, USA).

\section{Results}

A high treatment success rate (88.0\%) was obtained: the treatment was successful in 22 out of 25 cases (successful treatment group). Three patients underwent two-stage revision arthroplasty following ODPE due to treatment failure (treatment failure group), and other complications were not found in them. In knees with Tsukayma type II infection $(\mathrm{n}=10)$, the treatment success rate was $100 \%$. In knees with Tsukayma type III infection $(\mathrm{n}=15)$, the procedure was successful in $80 \%(\mathrm{n}=12)$, and thus all treatment failures occurred in knees with type III infection. However, the difference in the treatment success rate between type II infection cases and type III infection cases was not statistically significant $(\mathrm{p}=0.253)$.

In the treatment failure group, periprosthetic infection in the ipsilateral foot was found in one case and cervical parotid abscess and masticator space abscess were detected in another case (Figs. 2 and 3). No accompanying infection was found in the successful treatment group. Although the treatment failure group was small, the difference in the incidence of infection between the groups was statistically significant in the Fisher exact test $(\mathrm{p}=0.010)$. Heterogeneous materials included a constrained type component, rod, and internal fixation material implanted for previous periprosthetic fracture in one case in the treatment failure group and a constrained type component with rod in one case in the
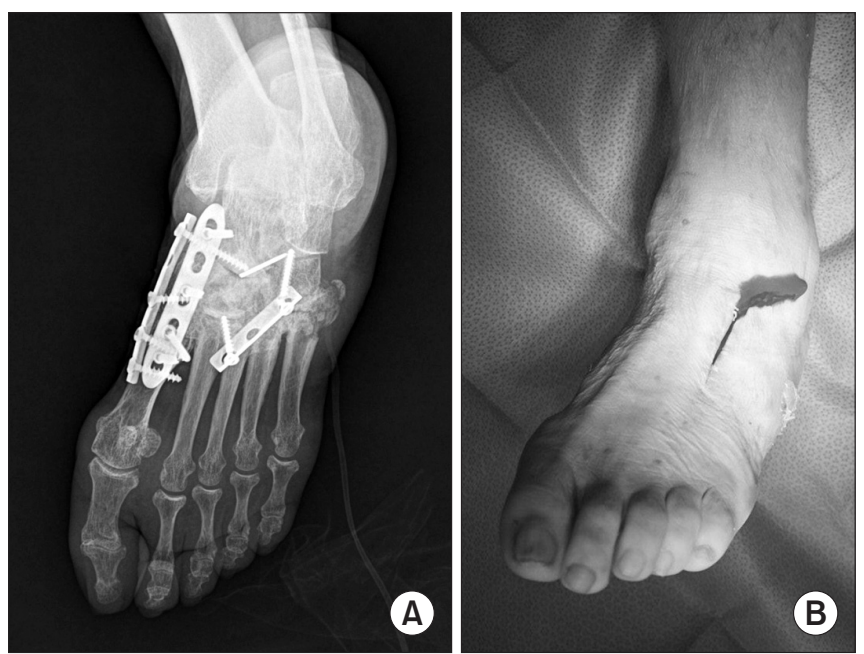

Fig. 2. Preoperative anteroposterior radiograph (A) and intraoperative photograph (B) showing periprosthetic infection in the ipsilateral foot in a treatment failure case. successful treatment group (Fig. 4), showing no statistically significant intergroup difference $(\mathrm{p}=0.230)$.

Bacterial culture results are presented in Table 2. Twelve cases were culture-negative, and four types of resistant bacteria were identified in the successful treatment group postoperatively, but no resistant bacteria were identified in the treatment failure group. There was no notable difference in the treatment success

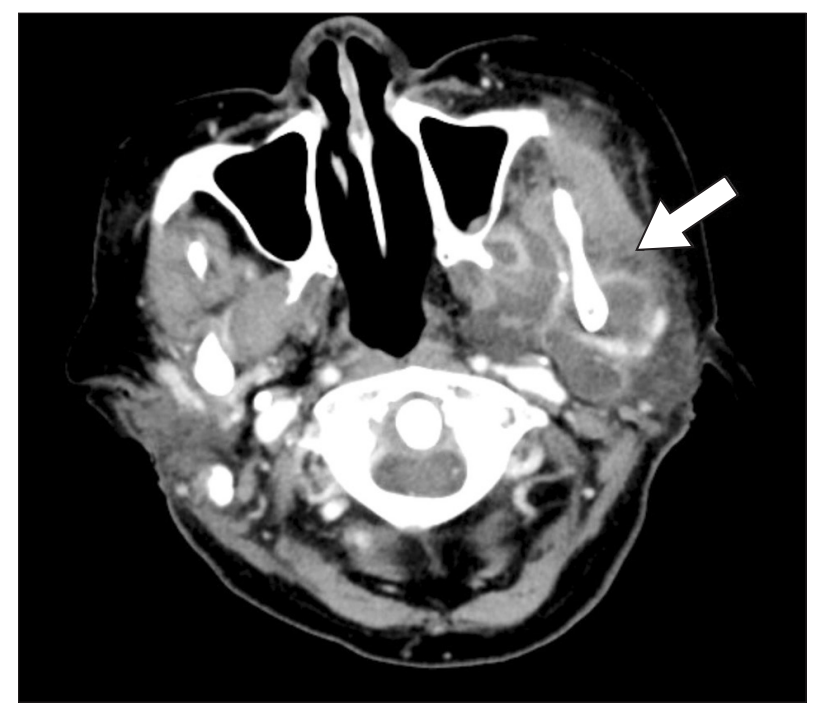

Fig. 3. Computed tomography of the neck showing accompanying cervical parotid abscess and masticator space abscess in a treatment failure case.
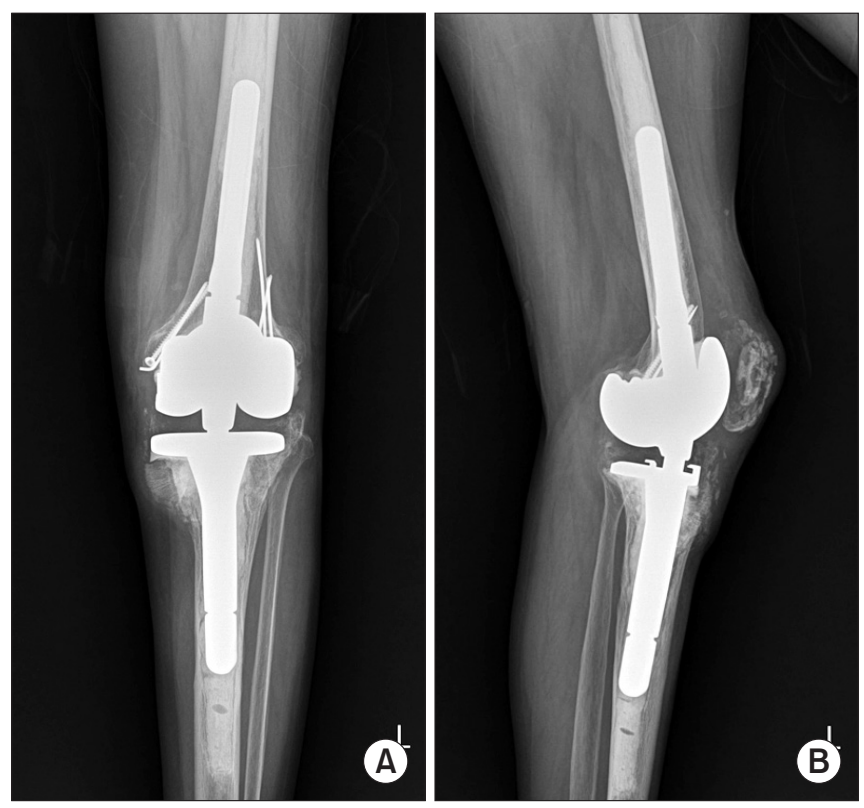

Fig. 4. Preoperative anteroposterior (A) and lateral (B) radiographs showing a constrained type component and internal fixation due to previous periprosthetic fracture in a treatment failure case. 
rate between infections caused by resistant bacteria and those caused by non-resistant bacteria ( $\mathrm{p}=1.002$ ) (Table 2 ).

TKA was performed due to RA in two of 22 cases in the successful treatment group and in two of three cases in the treatment failure group, showing a statistically significant intergroup difference ( $\mathrm{p}=0.048$ ). The patient age, ESR, CRP, synovial leukocyte count, and synovial neutrophil percentage showed no significant differences between groups (Table 3). In logistic regression analysis, there were no factors associated with treatment failure. In the successful treatment group, the mean Lysholm score was 81.4, and the mean KKS was 79.4, but the mean socio-emotional score of KKS was low with 18.8 points. The mean range of motion of the knee joint was $115.4^{\circ} \pm 12.9^{\circ}$, and the mean duration of hospitalization was $32.3 \pm 8.4$ days. Radiographic assessment showed $3.47 \pm 1.56 \mathrm{~mm}$ elevation of the joint line and a valgus change of $0.61^{\circ} \pm 2.35^{\circ}$ in knee alignment.

Table 2. Identified Bacteria in Both Groups

\begin{tabular}{ccccc}
\hline \multicolumn{2}{c}{ Treatment success group } & & \multicolumn{2}{c}{ Treatment failure group } \\
\cline { 1 - 2 } \cline { 4 - 5 } Identified bacteria & Case & & Identified bacteria & Case \\
\hline MRSA & 1 & & MSSA & 1 \\
MRSE & 2 & & Streptococcus & 1 \\
IRAB & 1 & & Enterobacter & 1 \\
MSSA & 1 & & \\
Polymicrobial & 1 & & \\
Streptococcus & 4 & & \\
No growth & 12 & & \\
\hline
\end{tabular}

MRSA: methicillin-resistant Staphylococcus aureus, MSSA: methicillinsensitive Staphylococcus aureus, MRSE: methicillin-resistance Streptococcus epidermidis, IRAB: imipenem-resistant Acinetobacter baumannii.

\section{Discussion}

For the treatment of infection after TKA, two-stage revision arthroplasty has been widely performed as an optimal standardized method yielding a success rate ranging from $85 \%$ to $95 \%{ }^{14,15)}$. However, Masters et al. ${ }^{16)}$ reported soft tissue stiffness and decreased mobility during the period between the two procedures. Hart and Jones ${ }^{17)}$ observed a long duration of hospitalization and antibiotic therapy following two-stage revision arthroplasty. Oduwole et al. ${ }^{18)}$ reported a heavy financial burden of the two-stage revision procedure. Although ODPE is reportedly less successful (success rate, $57 \%-75 \%)^{10}$, it is less likely to cause soft tissue stiffness and decreased mobility, requires a shorter duration of hospitalization and antibiotic therapy, and causes less financial burden than one- or two-stage revision arthroplasty ${ }^{18}$. Therefore, if ODPE can be successfully performed on selected patients with proper indications, it will be beneficial for the treatment of infection after TKA in many respects.

In this study, ODPE showed a high treatment success rate of $88 \%$. Despite the low socio-emotional scores, satisfactory results were obtained in terms of the KKS and Lysholm scores and range of motion of the knee joint. In addition, duration of hospitalization was short, and good radiographic results were obtained.

Based on the research results, we propose the following principles for ODPE. First, ODPE should be performed within 'five days' after the onset of symptoms before definite biofilm formation. In an in vivo study of the silastic subdermal implant colonized with Pseudomonas aeruginosa, it was reported that biofilm was enclosed in a fibrous capsule at week $4^{19)}$. Similarly, biofilm infection was observed at week 4 in an in vivo study of the Dacron graft colonized with Staphylococcus epidermidis ${ }^{20)}$. However,

Table 3. Comparison of Possible Associated Factors between Treatment Success group and Treatment Failure Group

\begin{tabular}{|c|c|c|c|}
\hline Variable & Treatment success group (22 cases) & Treatment failure group ( 3 cases) & p-value \\
\hline Age (yr) & $71.0(35-86)$ & $70.7(63-76)$ & 0.335 \\
\hline $\operatorname{ESR}(\mathrm{mm} / \mathrm{h})$ & $96.0(44-120)$ & $85.2(40-103)$ & 1.000 \\
\hline Synovial leukocyte count $(/ \mu \mathrm{L})$ & $58,663(1,750-328,000)$ & $67,700(1,800-150,000)$ & 0.089 \\
\hline Synovial neutrophil percentage (\%) & $90.38(60.00-100.00)$ & $85.75(80.00-90.00)$ & 0.115 \\
\hline Amount of foreign material & 1 (constrained type component) & $\begin{array}{l}1 \text { (constrained type component and internal } \\
\text { fixation due to previous periprosthetic fracture) }\end{array}$ & 0.230 \\
\hline Rheumatoid arthritis & 2 & 2 & 0.048 \\
\hline Resistant bacteria & 4 & 0 & 1.000 \\
\hline
\end{tabular}

ESR: erythrocyte sedimentation rate, CRP: C-reactive protein. 
considering that the most common bacterial strain in infection following orthopedic arthroplasty is more virulent Staphylococcus aureus, which stabilizes and enhances biofilm construction on the corrosion product of metal implant surfaces, it is difficult to apply those results to orthopedic arthroplasty ${ }^{21)}$. Recently, in 2015, Jorgensen et al. ${ }^{22)}$ conducted an in vivo study of biofilm formation of Staphylococcus aureus on the pin implant and reported that inconclusive biofilm clusters started to be observed on day 4 after implantation. They consistently found biofilms colonized on pins from day 6 onwards. Thus, they questioned the conventional definition of early infection, defined as infection occurring within three weeks. Koh et al. ${ }^{8)}$ reported that when the symptom duration was two weeks or longer, the success rate of ODPE was $20 \%$, and when it was one week or shorter, a high success rate of $78 \%$ was observed. In the current study, we obtained a high success rate of $80 \%$ in the case of Tsukayama type III infection treated within five days of symptom duration. Therefore, for Tsukayama type III infection, we think it is necessary to apply a stricter and more specific standard rather than the conventional extensive period of 'within three to four weeks after symptom onset. We used 'within five days after symptom onset, before biofilm formation, as the criterion, considering that a high success rate can be expected when surgical management and antibiotic therapy are performed before biofilm formation ${ }^{22-24)}$. Similarly, in Tsukayama type II infection, we recommend that ODPE be performed within five days from the onset of the infection symptom, not the usual postoperative symptom, rather than within four weeks after TKA. Even in the case of intraoperative or early postoperative infection, bacteria may have an occult period according to host immunity and pathogen virulence until they successfully reach and adhere to the implant, begin their programs for selfpreservation (transformation, communication, invasion production, and biofilm construction), and trigger an immune response with inflammation ${ }^{21)}$. Therefore, we suggest that better results of ODPE can be expected in Tsukayma type II if the procedure is performed within five days after the symptom onset, rather than being delayed until four weeks after TKA. In our study, we found that the symptom duration averaged 4.25 days (range, 3 to 9 days) in all successful treatment cases of type II infection. However, further research is necessary to validate our suggestion for using the symptom duration of five days as the indication for ODPE in type II infection. Furthermore, the conventional Tsukayma classification is believed to require a revision or subdivision considering the current situation where causative organisms acquire more virulence and resistance while immunocompromised older patients are increasing.
Second, preoperative examination to detect accompanying infection will contribute to more successful treatment of ODPE. Of the three treatment failure cases, two were accompanied by other infection (ipsilateral foot periprosthetic infection in one case and cervical parotid abscess and masticator space abscess in the other). The incidence of accompanying infection was statistically significantly high in the treatment failure group compared to that in the successful treatment group. Since other accompanying infections may be masked by TKA infection, they may be overlooked and remain unaddressed before ODPE. However, a failure to completely remove the infection source would increase the possibility of recurrence. This has not yet been established in the literature, and thus further research is warranted.

Third, although no statistical significance was found, it appears prudent to check the amount of heterogeneous substances before ODPE. In this regard, Holmberg et al..$^{7}$ reported a lower success rate when prosthetic materials and heterogeneous substances accompanied the procedure. In our study, one case out of total 22 cases in the successful treatment group had a constrained type. However, in the treatment failure group, since internal fixation was added, more heterogenous materials were involved due to the screw and K-wire, and we suspect that this could have affected treatment failure.

Fourth, TKA performed on RA patients raises the risk of failure of ODPE, so two stage revision arthroplasty should be considered as an option in such a case based on consultation with the patient. Lee et al. ${ }^{25}$ found through a meta-analysis that the deep infection rate and revision rate were significantly higher in patients who underwent TKA because of RA. In our study, we also found that RA was a significant failure factor.

Fifth, proper postoperative antibiotic therapy is expected to enhance treatment success. Vilchez et al. ${ }^{26)}$ reported that the use of rifampin increased the rate of treatment success when Staphylococcus aureus was detected. Holmberg et al. ${ }^{7}$ combined rifampin in the antibiotic therapy when Staphylococcus aureus was identified. Koh et al. ${ }^{8)}$ used isolated microorganism specific antibiotics with either rifampin or fluoroquinolone that targets biofilms. These studies showed high success rates $(75 \%$ and $71 \%$, respectively). Similarly, in our study, as soon as the growth of Gram-positive cocci was detected, rifampin and vancomycin were additionally used, and suitable antibiotics were chosen according to the bacteria isolated from synovial and wound culture. Therefore, we believe that the antibiotic therapy contributed to the treatment success in the two cases where Staphylococcus aureus was identified. In this regard, Vasso et al. ${ }^{27)}$ reported that when resistant bacteria are involved, treatment outcomes 
certainly appear less reliable, with increased possibility of treatment failure and higher rates of implant loss. In our study, when resistant bacteria were isolated, appropriate antibiotics which the bacterial strains are susceptible to were administered as soon as possible, and when it was determined on the basis of serial blood tests and clinical examinations that the patient did not respond to the antibiotics, the antibiotics were replaced with those which the bacteria are sensitive to. In the cases where methicillin-resistant Staphylococcus aureus isolation was successful in the treatment success group, vancomycin and rifampin were initially used, but there was no progress in the ESR and CRP blood test results for one week. Thus, the antibiotics were changed to linezolid, which was effective. However, further study is required to determine exactly when antibiotics should be changed or what degree of progress in the treatment indicates the need to change antibiotics. In this study, 12 (48\%) of the cases were culture-negative, which was relatively high considering that the incidence of negative culture in most infection series ranges from $0 \%$ to $25 \%{ }^{28,29}$. However, Kim et al. ${ }^{29)}$ reported that there was no difference between the culture-negative and culture-positive infection groups in terms of infection control and maintenance of functional status. Choi et al. ${ }^{30)}$ reported that a rather higher treatment success rate was obtained in the culture-negative infection group through the high dosage use of vancomycin and additional surgical treatment. In our patients, vancomycin and third-generation cephalosporin were extensively used in the case of culture-negative infection as well. Thus, although the large number of negative cultures does not appear to have affected the rate of treatment success or other results significantly, it has to be elucidated in further studies.

This study has some limitations. It is retrospective research involving a small number of patients in a single institution and the treatment failure group was relatively small, rendering statistical comparison difficult. It is, therefore, necessary to conduct a multicenter, prospective research in a larger number of patients. In addition, the follow-up period of some patients was only 24 months, which was short for assessing the possibility of recurrence. Last, this study included only the ODPE group, lacking evaluation of its usefulness through comparison with other procedures such as two-stage revision arthroplasty. In this regard, it is considered necessary to conduct further research including control groups in the future.

\section{Conclusions}

We recommend that ODPE be performed in combination with proper antibiotic therapy in selected patients with less than 'five days' of duration of symptom in the case of Tsukayma type III and Tsukayma type II infections. We also suggest that the following factors should be considered to obviate treatment failure, such as the presence of accompanying infection, diagnosis of RA prior to TKA, and the amount of heterogeneous substances. We believe that ODPE can be a useful method for successful infection treatment following TKA in addition to functional improvement, higher subjective satisfaction, and shorter hospitalization period.

\section{Conflict of Interest}

No potential conflict of interest relevant to this article was reported.

\section{References}

1. Kurtz SM, Lau E, Schmier J, Ong KL, Zhao K, Parvizi J. Infection burden for hip and knee arthroplasty in the United States. J Arthroplasty. 2008;23:984-91.

2. Peersman G, Laskin R, Davis J, Peterson M. Infection in total knee replacement: a retrospective review of 6489 total knee replacements. Clin Orthop Relat Res. 2001;(392):15-23.

3. Tsukayama DT, Goldberg VM, Kyle R. Diagnosis and management of infection after total knee arthroplasty. J Bone Joint Surg Am. 2003;85 Suppl 1:S75-80.

4. Lizaur-Utrilla A, Gonzalez-Parreno S, Gil-Guillen V, LopezPrats FA. Debridement with prosthesis retention and antibiotherapy vs. two-stage revision for periprosthetic knee infection within 3 months after arthroplasty: a case-control study. Clin Microbiol Infect. 2015;21:851.

5. Gardner J, Gioe TJ, Tatman P. Can this prosthesis be saved?: implant salvage attempts in infected primary TKA. Clin Orthop Relat Res. 2011;469:970-6.

6. Cobo J, Miguel LG, Euba G, Rodriguez D, Garcia-Lechuz JM, Riera M, Falgueras L, Palomino J, Benito N, del Toro MD, Pigrau C, Ariza J. Early prosthetic joint infection: outcomes with debridement and implant retention followed by antibiotic therapy. Clin Microbiol Infect. 2011;17:1632-7.

7. Holmberg A, Thorhallsdottir VG, Robertsson O, W-Dahl A, Stefansdottir A. 75\% success rate after open debridement, exchange of tibial insert, and antibiotics in knee prosthetic joint infections. Acta Orthop. 2015;86:457-62.

8. Koh IJ, Han SB, In Y, Oh KJ, Lee DH, Kim TK; Knee Multicenter Collaboration Team. Open debridement and prosthesis retention is a viable treatment option for acute peri- 
prosthetic joint infection after total knee arthroplasty. Arch Orthop Trauma Surg. 2015;135:847-55.

9. Schoifet SD, Morrey BF. Treatment of infection after total knee arthroplasty by debridement with retention of the components. J Bone Joint Surg Am. 1990;72:1383-90.

10. Deirmengian C, Greenbaum J, Lotke PA, Booth RE Jr, Lonner JH. Limited success with open debridement and retention of components in the treatment of acute Staphylococcus aureus infections after total knee arthroplasty. J Arthroplasty. 2003;18(7 Suppl 1):22-6.

11. Mont MA, Waldman B, Banerjee C, Pacheco IH, Hungerford DS. Multiple irrigation, debridement, and retention of components in infected total knee arthroplasty. J Arthroplasty. 1997;12:426-33.

12. Parvizi J, Zmistowski B, Berbari EF, Bauer TW, Springer BD, Della Valle CJ, Garvin KL, Mont MA, Wongworawat MD, Zalavras CG. New definition for periprosthetic joint infection: from the Workgroup of the Musculoskeletal Infection Society. Clin Orthop Relat Res. 2011;469:2992-4.

13. Classen T, Wegner A, von Knoch M. Modification of the method of Figgie for determination of joint line shifting in total knee arthroplasty. Radiologe. 2009;49:533-7.

14. Jamsen E, Stogiannidis I, Malmivaara A, Pajamaki J, Puolakka T, Konttinen YT. Outcome of prosthesis exchange for infected knee arthroplasty: the effect of treatment approach. Acta Orthop. 2009;80:67-77.

15. Volin SJ, Hinrichs SH, Garvin KL. Two-stage reimplantation of total joint infections: a comparison of resistant and nonresistant organisms. Clin Orthop Relat Res. 2004;(427):94100.

16. Masters JP, Smith NA, Foguet P, Reed M, Parsons H, Sprowson AP. A systematic review of the evidence for single stage and two stage revision of infected knee replacement. BMC Musculoskelet Disord. 2013;14:222.

17. Hart WJ, Jones RS. Two-stage revision of infected total knee replacements using articulating cement spacers and shortterm antibiotic therapy. J Bone Joint Surg Br. 2006;88:1011-5.

18. Oduwole KO, Molony DC, Walls RJ, Bashir SP, Mulhall KJ. Increasing financial burden of revision total knee arthroplasty. Knee Surg Sports Traumatol Arthrosc. 2010;18:945-8.

19. Buret A, Ward KH, Olson ME, Costerton JW. An in vivo model to study the pathobiology of infectious biofilms on biomaterial surfaces. J Biomed Mater Res. 1991;25:865-74.

20. Bandyk DF, Kinney EV, Riefsnyder TI, Kelly H, Towne JB.
Treatment of bacteria-biofilm graft infection by in situ replacement in normal and immune-deficient states. J Vasc Surg. 1993;18:398-405.

21. Gallo J, Kolar M, Novotny R, Rihakova P, Ticha V. Pathogenesis of prosthesis-related infection. Biomed Pap Med Fac Univ Palacky Olomouc Czech Repub. 2003;147:27-35.

22. Jorgensen NP, Meyer RL, Dagnaes-Hansen F, Fuursted K, Petersen E. A modified chronic infection model for testing treatment of Staphylococcus aureus biofilms on implants. PLoS One. 2014;9:e103688.

23. Nishitani K, Sutipornpalangkul W, de Mesy Bentley KL, Varrone JJ, Bello-Irizarry SN, Ito H, Matsuda S, Kates SL, Daiss JL, Schwarz EM. Quantifying the natural history of biofilm formation in vivo during the establishment of chronic implant-associated Staphylococcus aureus osteomyelitis in mice to identify critical pathogen and host factors. J Orthop Res. 2015;33:1311-9.

24. Jacqueline C, Caillon J. Impact of bacterial biofilm on the treatment of prosthetic joint infections. J Antimicrob Chemother. 2014;69 Suppl 1:i37-40.

25. Lee DK, Kim HJ, Cho IY, Lee DH. Infection and revision rates following primary total knee arthroplasty in patients with rheumatoid arthritis versus osteoarthritis: a metaanalysis. Knee Surg Sports Traumatol Arthrosc. 2016 Sep 7 [Epub]. http://dx.doi.org/10.1007/s00167-016-4306-8.

26. Vilchez F, Martinez-Pastor JC, Garcia-Ramiro S, Bori G, Macule F, Sierra J, Font L, Mensa J, Soriano A. Outcome and predictors of treatment failure in early post-surgical prosthetic joint infections due to Staphylococcus aureus treated with debridement. Clin Microbiol Infect. 2011;17:439-44.

27. Vasso M, Schiavone Panni A, De Martino I, Gasparini G. Prosthetic knee infection by resistant bacteria: the worstcase scenario. Knee Surg Sports Traumatol Arthrosc. 2016; 24:3140-6.

28. Berbari EF, Marculescu C, Sia I, Lahr BD, Hanssen AD, Steckelberg JM, Gullerud R, Osmon DR. Culture-negative prosthetic joint infection. Clin Infect Dis. 2007;45:1113-9.

29. Kim YH, Park JW, Kim JS, Kim DJ. The outcome of infected total knee arthroplasty: culture-positive versus culturenegative. Arch Orthop Trauma Surg. 2015;135:1459-67.

30. Choi HR, Kwon YM, Freiberg AA, Nelson SB, Malchau H. Periprosthetic joint infection with negative culture results: clinical characteristics and treatment outcome. J Arthroplasty. 2013;28:899-903. 